\title{
Erratum to: Exhaled breath online measurement for cervical cancer patients and healthy subjects by proton transfer reaction mass spectrometry
}

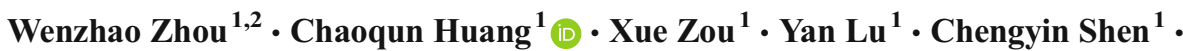 \\ Xiping Ding ${ }^{3} \cdot$ Hongzhi Wang ${ }^{1,3} \cdot$ Haihe Jiang ${ }^{1}$ Yannan Chu ${ }^{1}$
}

Received: 8 September 2017 / Accepted: 8 September 2017 /Published online: 25 September 2017

(C) Springer-Verlag GmbH Germany 2017

\section{Erratum to: Anal Bioanal Chem}

https://doi.org/10.1007/s00216-017-0498-0

The authors would like to call the reader's attention to the fact that unfortunately there was a mistake in the fund number in the acknowledgement section of the original article. The correct number is given below:

Acknowledgements This research was funded by the National Natural Science Foundation of China (Nos. 81401756, 21477132, 21107112) and National Key Technology Research and Development Program of the Ministry of Science and Technology of China (2015BAI01B04, 2013BAH14F01).

The online version of the original article can be found at https://doi.org/ 10.1007/s00216-017-0498-0

Chaoqun Huang

cqhuang@aiofm.ac.cn

$\triangle$ Hongzhi Wang

wanghz@hfcas.ac.cn

$\triangle$ Yannan Chu

ychu@aiofm.ac.cn

1 Anhui Province Key Laboratory of Medical Physics and Technology, Center of Medical Physics and Technology, Hefei Institutes of Physical Science, Chinese Academy of Sciences, 350 Shushanhu Road, Hefei, Anhui 230031, China

2 University of Science and Technology of China, No.96 Jinzhai Road, Hefei, Anhui 230026, China

3 Cancer Hospital, Chinese Academy of Science, 350 Shushanhu Road, Hefei, Anhui 230031, China 